\title{
Compressed sensing-based channel estimation for ACO-OFDM visible light communications in $5 \mathrm{G}$ systems
}

\author{
Muhammad Tabish Niaz ${ }^{1}$ Fatima Imdad', Waleed Ejaz ${ }^{2}$ and Hyung Seok Kim*
}

\begin{abstract}
In this paper, we propose a compressive sensing (CS)-based channel estimation technique for asymmetrically clipped optical-orthogonal frequency division multiplexing (ACO-OFDM) visible light communications (VLC) in 5G systems. We proposed a modified version of sparsity adaptive matching pursuit (SAMP) algorithm which is named as self-aware step size sparsity adaptive matching pursuit (SS-SAMP) algorithm. It utilizes the built-in features of SAMP and with additional ability to select step size according to the present situation, hence term self-aware, can provide better accuracy and low computational cost. It also does not require any prior knowledge of the sparsity of the signal which makes it self-sufficient. CS-based algorithms such as orthogonal matching pursuit (OMP), SAMP, and our proposed SS-SAMP were implemented on ACO-OFDM VLC. The paper is supported by simulation results which demonstrate performance of proposed scheme in terms of bit error rate (BER), mean square error (MSE), computational complexity, and key VLC parameter (LED nonlinearity, shot noise, thermal noise, channel response, and peak-to-average power ratio (PAPR). It is shown that the SS-SAMP is a good candidate for ACO-OFDM-based VLC that are mobile and have limited processing power, based on its performance and computational complexity.
\end{abstract}

Keywords: Bit-error-rate, Channel estimation, Compressed sensing, Visible light communication

\section{Introduction}

Visible light communication (VLC) is a promising optical wireless communication (OWC) technology which is paving its way to reality very quickly [1]. VLC uses LED emitted light which fulfills the dual functionality of illuminance and data transmission. VLC has shown potential to be an integral part of upcoming $5 \mathrm{G}$ networks. The market is continuously pushing the limits of the network data rate and capacity; it is very difficult for the wireless communication industry to meet these demands. It is estimated that for $5 \mathrm{G}$ networks, there will be a 1000-fold increase in data traffic [2]. In order to meet these demands, $5 \mathrm{G}$ networks will have to rely on other more efficient technologies. It is highly likely that it will incorporate smaller cells (atto-cells), additional spectrum, energy efficient communication, and heterogeneous networks (HetNet) integration [3].

\footnotetext{
* Correspondence: hyungkim@sejong.ac.kr

1 Department of Information and Communication Engineering, Sejong

University, Gwangjin-gu, Seoul, Republic of Korea

Full list of author information is available at the end of the article
}

When compared to the traditional radio frequency (RF) wireless communication, VLC has many strong characteristics [4] such as the following:

- Spectrum of visible light is free.

- Light that cannot penetrate solid objects makes it secure for indoor transmission.

- It can be deployed wherever LEDs are installed [5].

- The signal-to-noise ratio (SNR) is usually high because of high illumination requirements.

The fact that VLC can provide high bandwidth density can help in solving the demand of high bandwidth problem faced by upcoming RF-based networks. VLC can resolve supply and demand issue in 5G networks. Considering the positive traits of VLC at its present state can be best suited as a supplemental technology to assist HetNets in 5G networks. These main features of VLC make it a promising supplementary technology for 5G systems; however, it comes with various new challenges which open new research topics [6, 7]. 
Recently, optical orthogonal frequency division multiplexing (OFDM) systems have gained a lot of popularity due to high bandwidth, power efficiency, flexibility, and use of license-free spectrum [8]. The optical OFDM works on the technique of intensity modulation and direct detection (IM/DD) [9]. There are many proposed methods utilizing the optical OFDM in VLC systems [10-15]. In [11], Armstrong and Lowery have proposed asymmetrically clipped optical OFDM (ACO-OFDM) which is a very efficient technique to be used with VLC system. Instead of sending all subcarriers, ACO-OFDM sends only odd subcarriers. ACO-OFDM has been shown to be more efficient in terms of optical power than the systems that use DC-biasing as it utilizes a large dynamic range of the LED. The ACO-OFDM technique performs well under interferences caused by inter-symbol interference (ISI) and inter-carrier interference (ICI). It requires less optical power for a given data rate than other variants of optical OFDM if the constellation size is lower than 1024-QAM. In addition, ACO-OFDM is a good candidate for dimming systems because of its better performance at low-SNR regime. Due to these features, ACO-OFDM is used in this paper.

In VLC systems, due to interference from ambient light sources, estimating the channel for correct signal recovery is of utmost importance. Among studies on ACO-OFDM VLC systems, there have been proposals for channel estimation, which is an important part of wireless communication systems. In [12], authors have proposed a technique to find channel state information (CSI) in the ACO-OFDM communication system for better channel estimation. A study has proposed a least square (LS)-based channel estimator to achieve highly accurate result in ACO-OFDM VLC systems [13]. Another study proposed the use of minimum mean square error (MMSE) and least minimum mean square error (LMMSE) based methods to improve the channel estimation of ACO-OFDM VLC systems $[14,15]$. There is an alternate approach for channel estimation, which utilizes the technique of compressed sensing (CS). The CS-based technique exploits the signal sparsity and incoherency to achieve best results [16-20]. It relies on finding sparse solutions to underdetermined linear systems and can estimate signals from fewer samples rather than using the Nyquist sampling rate [21]. The positive aspect of using CS technology is to have more accurate, fast, and reliable channel estimation techniques, which are inexpensive and have low complexity.

\section{Related work}

The CS algorithms, which recover a sparse signal, are generally divided into two branches: linear programming (LP) and dynamic programming (DP). This paper focuses on the DP algorithm because most of the LP algorithms tend to have high computational complexity. Due to the high complexity of LP algorithms, they do not perform well in real large-scale applications. The orthogonal matching pursuit (OMP) algorithm [22] is by far the most popular algorithm in DP category [23, 24]. The major drawback in DP algorithms is that they rely on the knowledge of channel sparsity beforehand, which is not always possible in real practical applications. To counter the problem of channel sparsity information, sparsity adaptive matching pursuit (SAMP) was proposed in [25]. The SAMP algorithm uses an iterative method to estimate the signal sparsity, with a fixed step size to be used in each stage. This was an improvement over OMP algorithm, which usually requires the level of sparsity as a priori information to estimate the original signal. The research in [25] showed that SAMP outperforms OMP and its variants, but its drawback is that the MSE performance and complexity are directly related to the initial step size. Since mobile devices equipped with VLC modules have limited resources, the high computational complexity is a huge disadvantage.

There is no such literature that has worked on CS-based channel estimation in ACO-OFDM VLC systems. To the best of our knowledge, one article claims to improve the channel bandwidth of simple OFDM VLC system using CS [26]. The authors propose a new technique that can compensate the lack of channel bandwidth in an optical-OFDM link by using compressive sensing. The positive aspect of using CS technology is to have more accurate, fast, and reliable channel estimation techniques that are inexpensive and have low complexity.

To summarize the current trend of research, most of the CS-based techniques are devised for RF-based channel estimation. CS is very new in VLC domain, and there are very few literatures available which makes it a very open field for research.

\section{Contribution}

In this paper, a new self-aware step size SAMP (SS-SAMP) CS-based channel estimation algorithm is proposed for ACO-OFDM VLC system. The main contribution of our work is as follows:

- SS-SAMP can adaptively adjust the step size.

- Due to the adaptive adjustment of step size, convergence is faster.

- SS-SAMP does not require a priori knowledge of sparsity as in the case of other mentioned algorithms.

The paper is organized as follows: Section 2 describes the system model used in the paper; Section 3 presents the proposed CS-based channel estimation techniques; Section 4 discusses the effect of various VLC key parameter impact; 
Section 5 presents simulation for the performance comparison with existing CS-based channel estimation algorithms; finally, Section 6 concludes the paper.

\section{System model}

The block diagram of ACO-OFDM system is shown in Fig. 1. The system has three main parts: transmitter, channel, and receiver. The transmitter is made up of OFDM modulator and optical modulator. The channel is assumed as a generic optical wireless channel. The receiver again is divided into two main parts: the optical demodulator and OFDM demodulator.

The main requirement of a VLC system to work is to have real and positive values from the optical modulator. This property usually employs the presence of IM/DD technique. ACO-OFDM also works on the IM/DD technique and thus is simple to implement. The main problem here lies with the OFDM signal. Since they are complex and bipolar, simple OFDM system cannot be used with VLC system. It is necessary to make it unipolar and real. The ACO-OFDM modulator inherently does this work.

To ensure that the output of ACO-OFDM is real and unipolar, the following procedure is followed [14]. There are $N$ sub-carriers out of which only odd ones will be used to send data. At the transmitter, the source bits are first modulated using Gray-coded M-ary quadrature amplitude modulation (QAM) mapping block. This will produce a complex bit stream. Then to maintain real OFDM symbols in time domain, the encoded data is passed through the Hermitian symmetry block. The data is then passed on to inverse fast Fourier transform (IFFT) block and clipping block so that the data is on odd subcarriers and positive.
Cyclic prefix (CP) is later added to avoid ISI. The addition of CP has a negligible impact on the SNR and spectral efficiency of the VLC system. To reduce the peak-toaverage power ratio (PAPR), clipping is performed to combat two types of distortions: firstly the out-of-band distortion which is reduced by applying filtering and secondly the in-band distortion which is reduced by adding the CP. It is to be noted that in ACO-OFDM signal, only odd sub-carriers are sent [14]. The signal is converted from analog to digital using an analog-todigital converter (ADC) and later, using optical intensity modulation (IM), the data is sent over the optical wireless channel.

There are two more types of interference noises, the narrowband interference (NBI) and impulse interference, which have to be taken into consideration. To solve these interferences, there are two methods that are commonly used in ultra-wideband communication systems. One method is based on frequency domain and the other is based on time-domain schemes [27]. While keeping strictly within the scope of this paper, the frequency domain will be discussed. For frequency-domain schemes, OFDM is used to combat these interference noises. One of the most prominent methods is the frequency threshold excision (FTE), in which the excess interference is clipped from the signal. The advantage of using ACO-OFDM is the intrinsic immunity to interferences like NBI. It is shown in [11] that the interference component lies in the even sub-carriers while the odd sub-carrier's components are affected by the multiplication of a constant 0.5. ACO-OFDM also mitigates these interferences by clipping the signal before transmission.

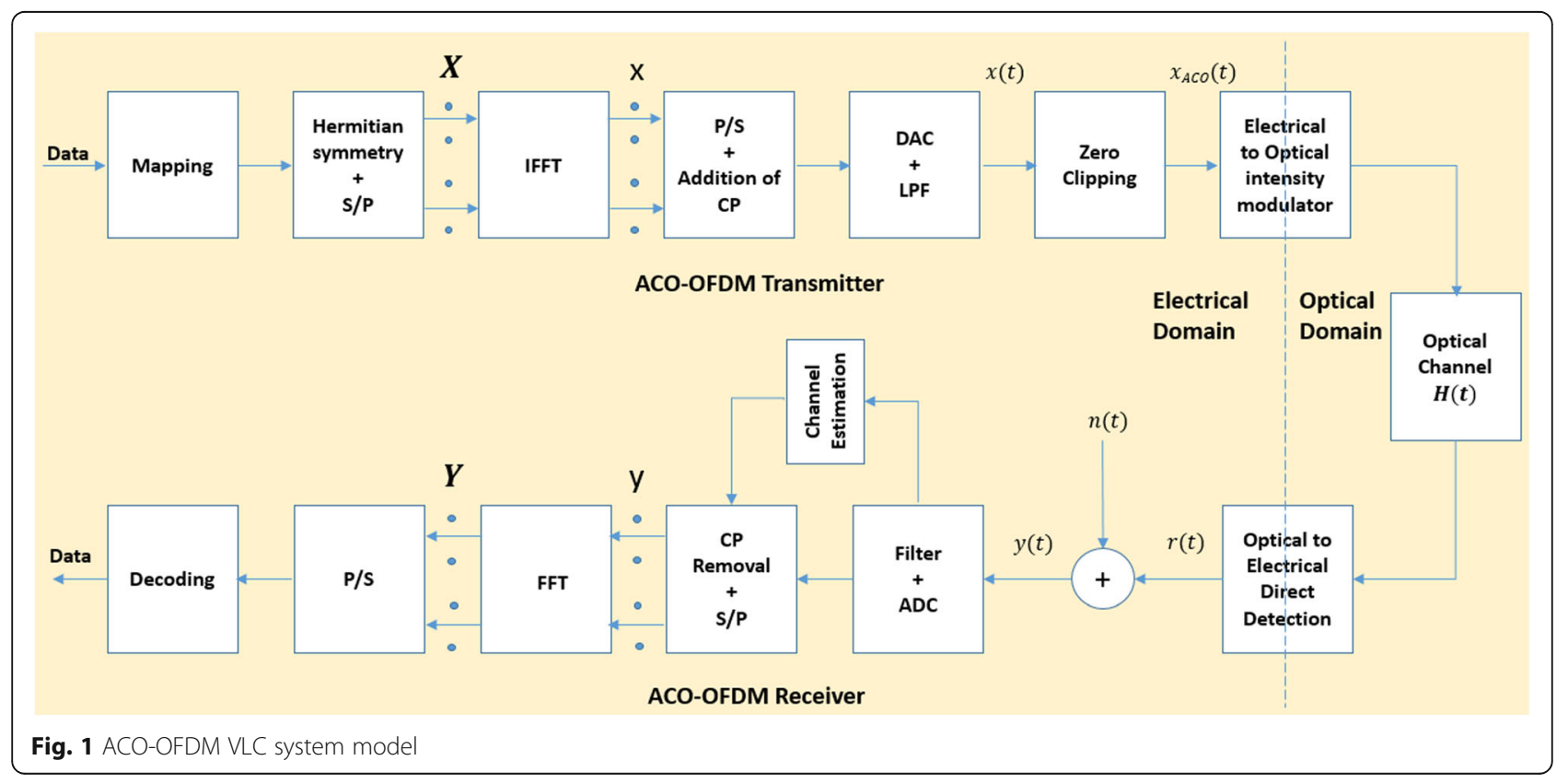


This helps in containing the signal within the time frame hence minimize the interference. To further reduce the effects of these interferences, a narrowband optical filter can be used at the receiver [28-32]. CS has many applications, one of these applications is of channel estimation, which is discussed in this paper. One of the other applications of CS is the noise cancellation in which the sparsity is used to mitigate the effect of interference noises including NBI and impulse noise. This discussion is out of the scope of this paper, since the scheme used in this paper strictly deals with the channel estimation. The intrinsic immunity of ACO-OFDM towards the interference noise tries to minimize the effect of these interferences.

At the receiver's end, using direct detection (DD), the signal is received using photodiode (PD). This will convert the signal to digital electrical domain. The received signal is first recovered using different CS channel estimation techniques. The estimated signal is then passed to the ACO-OFDM demodulator block. After demodulation, the signal goes through the demapping block and original data stream is recovered.

The received signal at the receiver in time domain is represented as follows:

$$
y(t)=h(t) x_{\text {aco }}(t)+n(t)
$$

In VLC, the channel $h$ can be line-of-sight (LOS) and non-line-of-sight (NLOS). The main property of $h$ is that it is always real and positive. The received signal in frequency domain can be expressed as

$$
Y=H X_{\text {aco }}+N
$$

where $X_{\text {aco }}$ is the ACO-OFDM signal, $H$ is the OWC channel response, and $N$ is the noise. In this case, to make things simple additive white Gaussian noise (AWGN) is assumed. In optical channels, the quality of transmission is typically dominated by shot noise [33]. The desired signals contain a time-varying shot-noise process which has an average rate of $10^{4}$ to $10^{5}$ photons/ bit. Since in an office/home environment, there is ambient light striking the detector which leads to a steady shot noise having a rate of the order of $10^{7}$ to $10^{8}$ photons/bit, even if the receiver employs a narrowband optical filter. Therefore, in optical channels the ambient shot noise is modeled as a Gaussian process [34], hence AWGN channel is assumed. This is further discussed in detail in Section 4.1. Since VLC is considered to be an ultra-wideband (UWB) communication system [35], where the multipath components of the signal arrive at time intervals that are larger than the inverse of the bandwidth of the considered channel. The sparsity depends on two aspects: the considered bandwidth (the larger the bandwidth the more likely the channel is sparse) and the considered environment. Since VLC has a large bandwidth, the channel vector can be well approximated as a sparse signal [36-40]. Even if the desired channel vector is not sparse, the CS theory allows one to choose proper basis to express the signal as a linear combination of basis. Perfect synchronization between the transmitter and receiver is assumed.

Considering the two types of optical channel (i) LOS and (ii) NLOS, the expression of both types of channel can be found from Eqs. (3) and (4). The total diffused channel response can be computed by Eq. (6).

$$
\begin{aligned}
& H_{\text {los }}(0) \\
& =\left\{\begin{aligned}
\frac{A_{r}\left(m_{1}+1\right)}{2 \pi d^{2}} \cos ^{m_{1}}(\phi) T_{s}(\psi) g(\psi) \cos (\psi), & \mid 0 \leq \psi \leq \psi_{C} \\
0, & \text { elsewhere }
\end{aligned}\right. \\
& \begin{aligned}
H_{\text {nlos }}(t)= & \frac{\left(m_{1}+1\right)}{2 \pi} \sum_{j=1}^{K} \rho_{j} \cos ^{m_{1}}\left(\phi_{j}\right) \frac{\cos (\psi)}{d_{j}^{2}} \operatorname{rect}\left(\frac{2 \psi}{\pi}\right) \\
& \times H_{\text {nlos }}^{(k-1)}\left(t-\frac{d_{j}}{c}\right) \Delta A
\end{aligned} \\
& H_{\text {nlos }}(t)=\sum_{i}^{K} \sum_{k=0}^{\infty} H_{\text {nlos }}^{k}(t) \\
& H_{\text {total }}(0)=H_{\text {los }}(0)+\sum_{\text {refl }} H_{\text {nlos }}(0)
\end{aligned}
$$

where $A_{r}$ is the photodetector area, $m_{1}$ is the Lambert's mode number expressing directivity of the source beam, $T_{s}(\psi)$ is the receiver with optical bandpass filter, $g(\psi)$ is the concentrator gain, $\psi$ is the radiation incident angle, $\phi$ is the angle with respect to transmitter, $d$ is the distance of the transmitter, $\Delta A$ is the area of reflecting elements, $k$ is the total number of reflector elements in the room, $\rho_{j}$ is the reflection coefficient of $j$, and $H_{\text {nlos }}^{(k-1)}$ is the impulse response of order $k-1$ between the reflector and receiver.

\section{CS-based channel estimation method}

In this section, the different channel estimation algorithms are discussed and the proposed scheme is given.

\subsection{Least square channel estimation}

The LS channel estimation is the simplest one. The only catch with this estimation is that the optimal solution is only achieved when there is no noise and interference is considered in the received signal. The mean square error (MSE) is high, hence accuracy is limited. Thus, concluding the LS estimation technique is not the best solution for this CS-based channel estimation. 


\subsection{OMP channel estimation algorithm}

One of the earliest greedy iterative algorithm is orthogonal matching pursuits (OMP). It is one of the main stepping stones on which the other greedy algorithm is based on. The basic working of an OMP algorithm revolves under the conditions of a given iteration number. Since it is an iterative process, it is required to stop after certain iterations. To make accurate estimation of the original signal, OMP requires a lot of measurement data. One of the drawbacks of the OMP is that, as it requires a large number of measurement data to estimate the signal, any increase in the degree of sparse or the number of samples would increase the time it takes to obtain the results. The basic working of OMP algorithm is shown in Algorithm 1.

\subsection{SAMP channel estimation algorithm}

The OMP algorithm is not adaptive. A pre-estimate of the sparse degree of the signal should be known and also the estimation accuracy is not so good. In an actual scenario, the sparse degree is not known; hence, to make things easier and less complex the adaptive algorithm like sparsity adaptive matching pursuit (SAMP) was proposed in [24]. With this adaptive algorithm, the signal estimation does not require any information of signal sparse degree beforehand. The working of SAMP requires to choose a step size $s$, which should satisfy $s \leq K$. The trade-off of choosing the step size is that if it is too small the algorithm will take long time to converge. Hence the choice of the step size is very important in this algorithm. The SAMP algorithm has a high computational complexity and more computational time than OMP algorithm. The basic working of SAMP algorithm is shown in Algorithm 2.

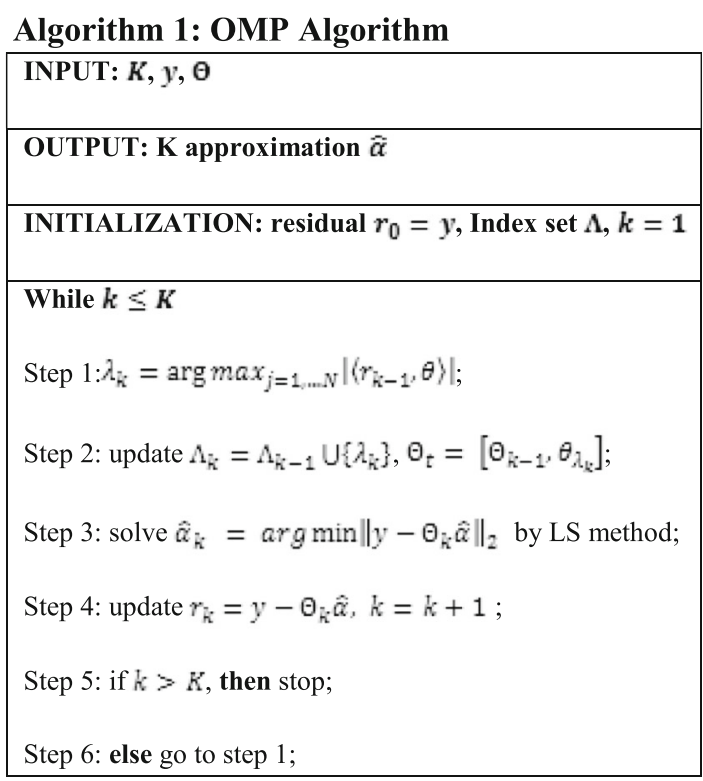

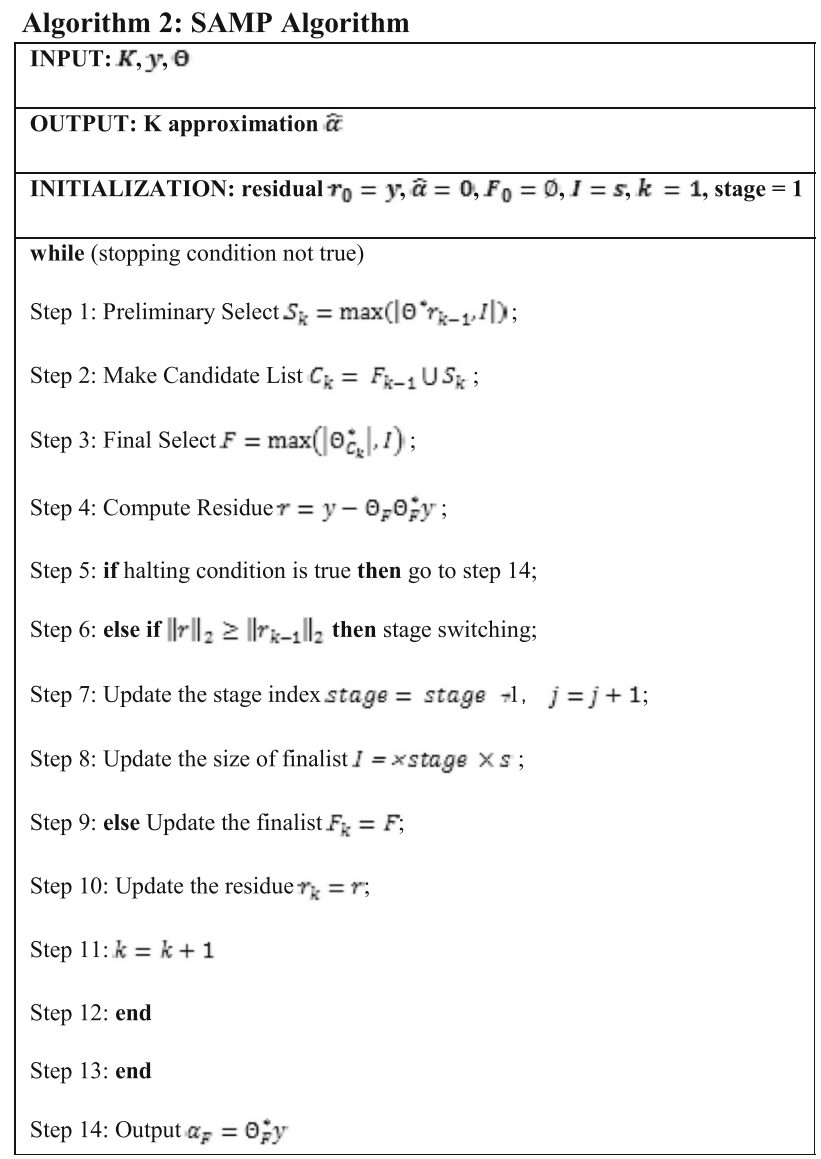

\subsection{SS-SAMP channel estimation algorithm}

The choice of step size is the main parameter which defines the performance of the proposed self-aware step size sparsity matching pursuit (SAMP) algorithm. If the chosen step size is small, SAMP algorithm leads to better estimation accuracy but increases the computational complexity. On the other hand, if the step size is large, the computational complexity decreases but at the cost of low estimation accuracy. By making the algorithm self-aware with respect to step size, a better trade-off can be maintained between the estimation accuracy and computational complexity. The self-aware step size in this particular case depends on the current state of signal estimation from the original signal. The current state of estimation can be the current estimated signal energy or the estimated sparsity of the current signal. Since the estimation of sparse elements is done with large values at the initial stages of the algorithm, the values are reduced as the later stages come. This means that the energy tends to stabilize as the estimated sparsity is close to true sparsity $K$. Utilizing this property of sparse signals, the proposed SS-SAMP algorithm starts by choosing step size which is large and, as the algorithm progresses, the energy of the signal decreases at a certain rate which defines by how much the step size will be reduced. 
To specify the fine-tuning process, an additional threshold $\gamma$ is specified. The proposed algorithm is shown in Algorithm 3. The algorithm is stagewise with a variable size of the final support set $F_{k}$ in different stages. During each stage, $F_{k}$ adapts to two correlation test. These tests are the candidate test and final test, which searched a certain number of coordinates corresponding to the largest correlation values between the signal residual and the columns of the measurement matrix. In the next stage the algorithm runs until the recovered signal is found which has the least residual. The SS-SAMP uses two threshold values for halting criterion. These two values are tolerance $\varepsilon$ and $\gamma$. The SSSAMP algorithm comes to a halt when the residual's norm is smaller than $\varepsilon$. On the other side, the values of the step size are decreased as the difference in energy of the estimated signal and original signal falls below $\gamma$.

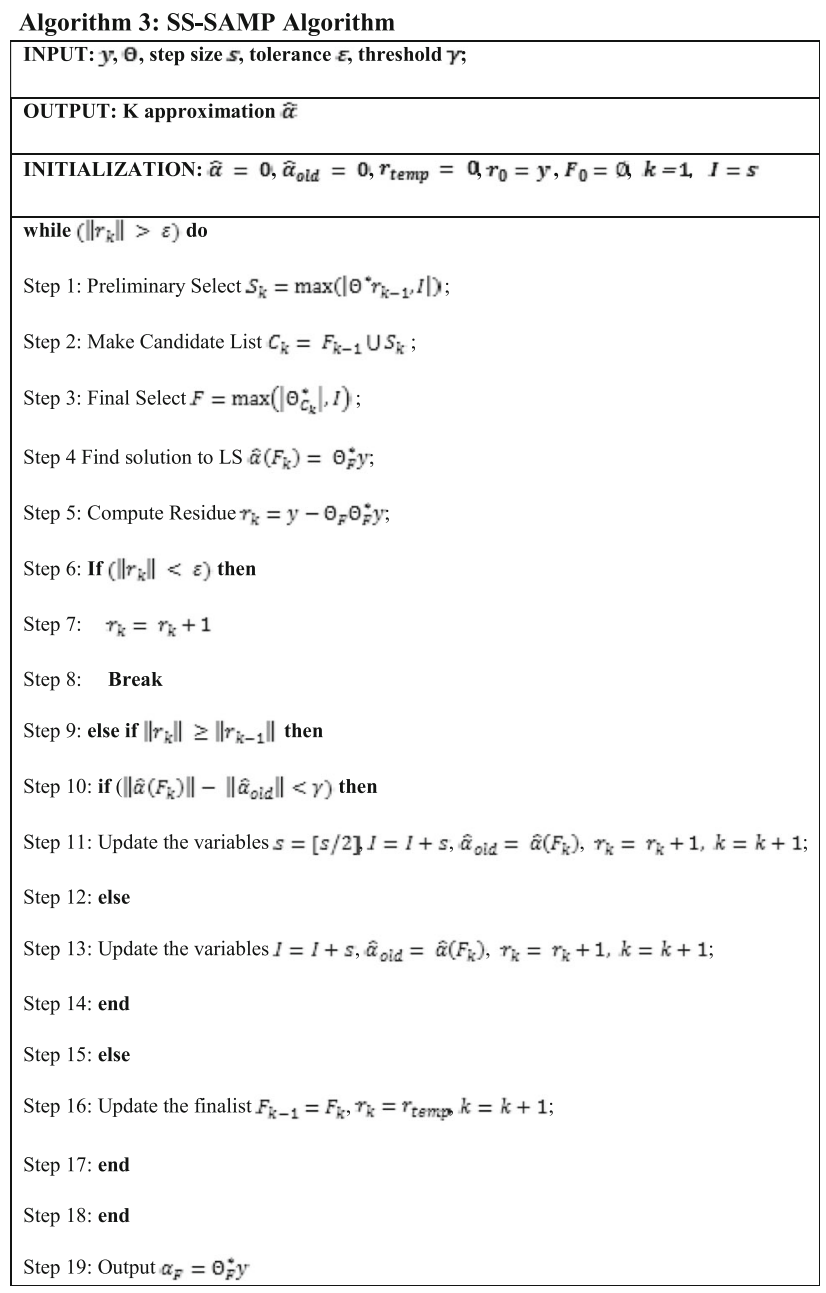

\section{Discussion on different VLC characteristics}

In this section, different VLC characteristics are discussed such as LED nonlinearity, shot noise, and PAPR calculation. The effects of the proposed scheme on these parameters are discussed, and the results are shown in Section 5.

\subsection{LED nonlinearity, shot noise, and thermal noise}

Since this paper utilizes ACO-OFDM as the modulation scheme, one drawback is that the signal is limited due to the constraints imposed by the dynamic range of the LED. Due to the central limit theorem, the time domain signal $x(k)$, depicts a Gaussian distribution for IFFT sizes larger than $N=64$ [41]. Therefore, according to [42], the average electrical symbol power is

$$
E_{s}=2 \sigma_{x(k)}^{2}
$$

where $\sigma_{x(t)}^{2}$ is the variance of the signal.

Due to the directly proportional relationship between the radiated optical power and the forward current of the LED, the signal and constraints are described in terms of optical power. There are two main points on concern in the dynamic range imposed by the LED. One is the minimum optical power point, termed as $P_{T x, \min }$, and the other point is the maximum optical power point, termed as $P_{T x, \max }$. The point at which the optical power bias is indicated is termed as $P_{T x, b i a s}$. The signal is clipped at the top level, to ensure the maximum power driving limit of the LED. The clipping at the top level can be expressed as $\epsilon_{\text {top }}=P_{T x, \max }-P_{T x, \min }$. If the LED is insufficiently forward biased, then it should be clipped at the bottom level, $\epsilon_{\text {bottom }}=P_{T x, \min }-P_{T x, \text { bias }}$, this condition will only hold true if $P_{T x, \text { bias }}<P_{T x, \text { min }}$. The signal after the conversion is then transmitted over the optical wireless channel $h$.

Since it is already mentioned that the optical power is directly proportional to the forward current in the LED, the relationship of the two variables is given below. In order to characterize the nonlinear distortion caused by the LED, the Bussgang theorem [41] is applied. The theorem states that the nonlinear distortion can be modeled as an attenuation of the transmitted signal plus a distortion noise component, $\eta_{\text {clip }}(k)$. The system is evaluated on the basis of the ratio between the power of the undistorted part of the signal and the effective noise power, called effective SNR, which accounts for the contributions caused by the clipping, shot noise, and thermal noise. The ratio is given as:

$$
\mathrm{SNR}=\frac{K^{2}(E[x(k)])^{2}}{\sigma_{\text {clip }}^{2}+\frac{\sigma_{n(k)}^{2}}{2 h^{2} \alpha^{2}}}
$$

where $\alpha$ is the responsivity of the PD detector and $E[x(k)]$ is the undistorted part of the signal power corresponding to the transmitted optical power of least signal clipping. The attenuation factor, $\mathrm{K}$, and the variance of the clipping noise $\sigma_{\text {clip }}^{2}$ are derived from [42]. The noise variance of the white Gaussian noise $\sigma_{n(k)}^{2}$ is the sum of the shot noise and the thermal noise. 


$$
\begin{aligned}
& \sigma_{n(k)}^{2}=\sigma_{\text {shot }}^{2}+\sigma_{\text {thermal }}^{2} \\
& \sigma_{\text {shot }}^{2}=2 q \alpha P_{r} B+2 q I_{b g} B \\
& \sigma_{\text {thermal }}^{2}=\frac{4 k T}{R_{L}} B
\end{aligned}
$$

where $q$ is the electronic charge, $I_{b g}$ is the background light current, $B$ is the bandwidth, $P_{r}$ is the optical power received, $T$ is the absolute temperature, $R_{L}$ is the load resistance, and $k$ is the Boltzmann's constant.

The analysis of the nonlinearity is done in Section 5.

\subsection{PAPR}

In this section the peak-to-average power ratio (PAPR) of the ACO-OFDM is discussed and the results will be analyzed in Section 5. The PAPR is defined as the maximum power of the transmitted signal divided by the average power. The PAPR is given as:

$$
\mathrm{PAPR}=\frac{\max x^{2}(k)}{E\left[x^{2}(k)\right]}
$$

Since in OFDM, there is a large number of sub-carriers, the system has a large dynamic signal range and exhibit a very high PAPR. Later when this OFDM signal is passed through the nonlinear LED, the signal degrades further and that effects the overall BER performance.

PAPR is usually presented in the form of CCDF. In this, we find the probability that PAPR value is higher than a certain PAPR value $\mathrm{PAPR}_{0}$, i.e., $P_{r}\left(\mathrm{PAPR}>\mathrm{PAPR}_{0}\right)$. The simulation results are analyzed in Section 5.

\section{Performance evaluation}

In this section, the performance of different channel estimation techniques is simulated and later the proposed scheme performance is analyzed on the basis of the key VLC parameters. The comparison of LS, OMP, SAMP, and proposed SS-SAMP are shown in two parameters, BER and MSE, since the main parameter that defines the accuracy of estimation signals is based on the step size. To simulate, three different step sizes are chosen: small, medium, and large step size. The MSE and BER are used to measure the channel estimation accuracy and the system performance, respectively. To measure the computational complexity, CPU running time was computed. Simulations were performed in MATLAB R2015a using the i5 CPU with $4 \mathrm{~GB}$ of memory. The results were averaged using 1000 Monte-Carlo trials. Later, the proposed scheme is analyzed on how it will tackle the LED nonlinearity, shot noise, and thermal noise. Also, the channel response and the modulation constellation are analyzed to give a clear picture on how the proposed scheme will perform in an indoor VLC environment.
A simple VLC system model is implemented with ACO-OFDM. Simulation parameters are shown in Table 1. The optical wireless channel model used in this simulation is based on the work proposed by [43]. The calculation of ACO-OFDM BER with a M-QAM constellation is given as:

$$
\text { BERACO-OFDM }_{\text {ACo }}=\frac{4}{\log _{2} M}\left(1-\frac{1}{\sqrt{M}}\right) \operatorname{erfc}\left(\sqrt{\frac{3 \mathrm{SNR}}{2(M-1)}}\right)
$$

The calculation of MSE is done using the following Eq. (14):

$$
\mathrm{MSE}=E\left[\sum_{k}|H(k)-\hat{H}(k)|^{2}\right]
$$

To evaluate the performance, BER was computed for all mentioned algorithms. Figure 2 shows that CSbased channel estimators perform better than the conventional LS-based estimator. It can be seen that the SS-SAMP performs better than SAMP. To further investigate the performance, the MSE is shown in Fig. 3. Again, the same trend can be seen as LS-based channel estimation algorithm performs poor with respect to the CS-based channel estimation algorithms. Since the step size is chosen according to the current

\begin{tabular}{|c|c|}
\hline Parameter & Value \\
\hline Room dimensions (m) & $5 \times 5 \times 3$ \\
\hline Height of receiving plane (m) & 0.8 \\
\hline Number of LEDs & 256 \\
\hline Center luminous intensity, $I_{0}$ (cd) & 30 \\
\hline Minimum optical power, $P_{T x, \min }(\mathrm{mW})$ & 90 \\
\hline Maximum optical power, $P_{T x, \max }(\mathrm{mW})$ & 400 \\
\hline Semiangle at half power $\left(^{\circ}\right)$ & 60 \\
\hline Photodiode responsivity & 0.3 \\
\hline Field of view at the receiver $\left(^{\circ}\right)$ & 85 \\
\hline Reflective index of concentrator & 1.5 \\
\hline$A_{r}\left(\mathrm{~cm}^{2}\right)$ & 1 \\
\hline Background noise current, Ibg $(m A)$ & 0.62 \\
\hline Load resistance, $R_{L}(\mathrm{~K} \Omega)$ & 10 \\
\hline Absolute temperature, $T\left({ }^{\circ} \mathrm{K}\right)$ & 295 \\
\hline Reflectivity, $\rho$ walls and floor & 0.8 and 0.3 \\
\hline Modulation & 64-QAM ACO-OFDM \\
\hline
\end{tabular}
status of the estimation signal, SS-SAMP outperforms SAMP and OMP.

To further investigate the performance of SAMP and SS-SAMP with respect to the step size, Fig. 4 shows the

Table 1 Simulation parameters

$m$ meter, $W$ watt, ${ }^{\circ}$ degree, $\mathrm{cm}^{2}$ square centimeter, $c d$ candela 


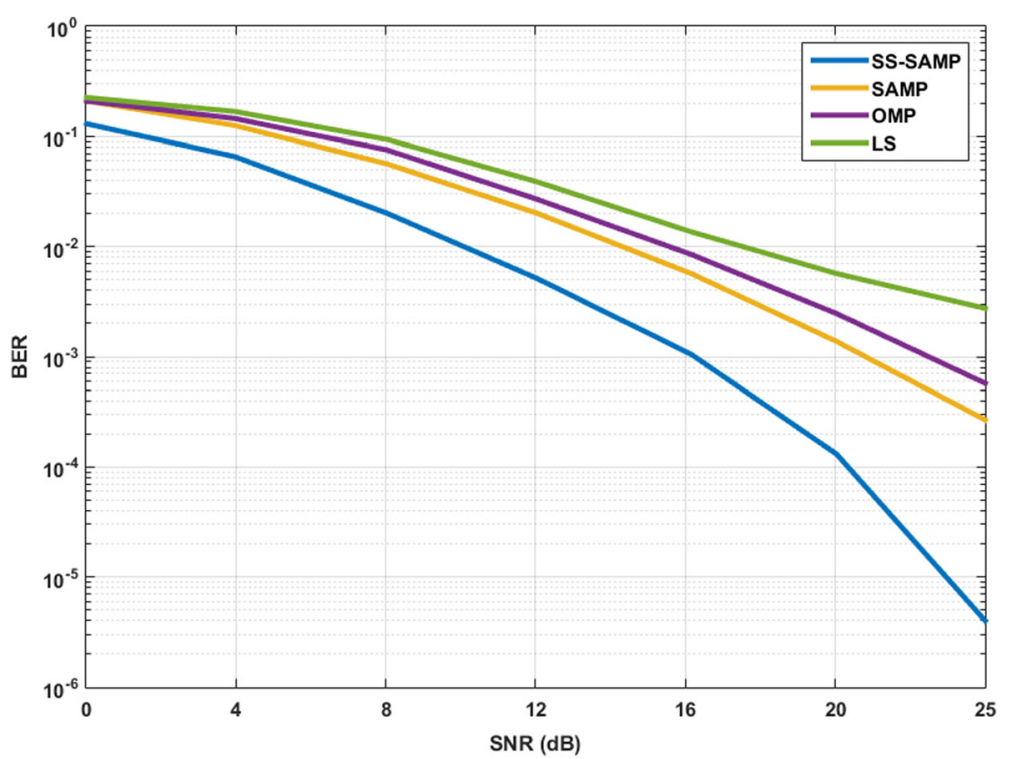

Fig. 2 BER performance of different CS channel estimation algorithms

performance of the two algorithms with three different initial step sizes. Earlier in Section 2, it was mentioned that the step size was chosen based on small, medium, and large values. These values are one for small, six for medium, and eight for large. Both algorithms are compared using these three step sizes, and Fig. 4 summarizes the results obtained. It can be seen that if small step size is chosen, the performance of both algorithms are the same. This is because, under this condition, both algorithms perform in the same way. But if we increase the step size to medium or large, SS-SAMP outperforms SAMP.
To conclude the performance analysis of the proposed SS-SAMP algorithm, running time of each CS-based channel estimation algorithm mentioned in this paper was computed to depict the computational complexity. All the algorithms were run on the same system with the same environment. Figure 5 shows the running time of these algorithms. From the results, it can be seen that OMP takes most of the time to run while SAMP, and SS-SAMP all give a respectable running time. It can be seen that as the SNR increases, both SAMP and SS-SAMP performances are comparable with a difference of approximately $0.002 \mathrm{~s}$. Looking at all the results

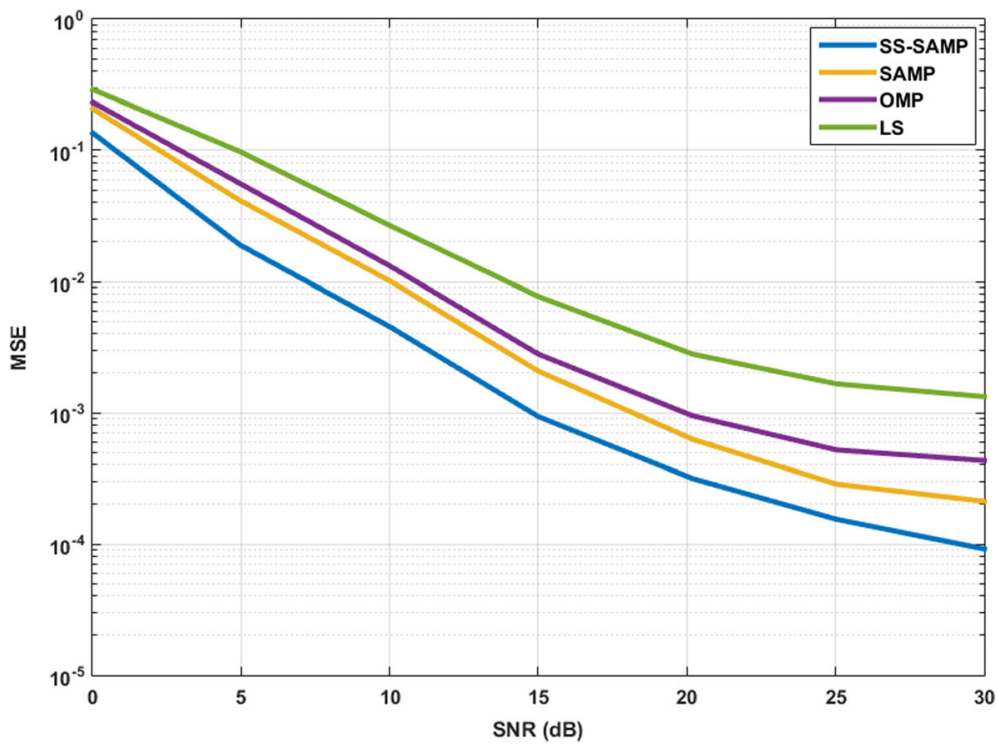

Fig. 3 MSE calculation of different CS channel estimation algorithms 


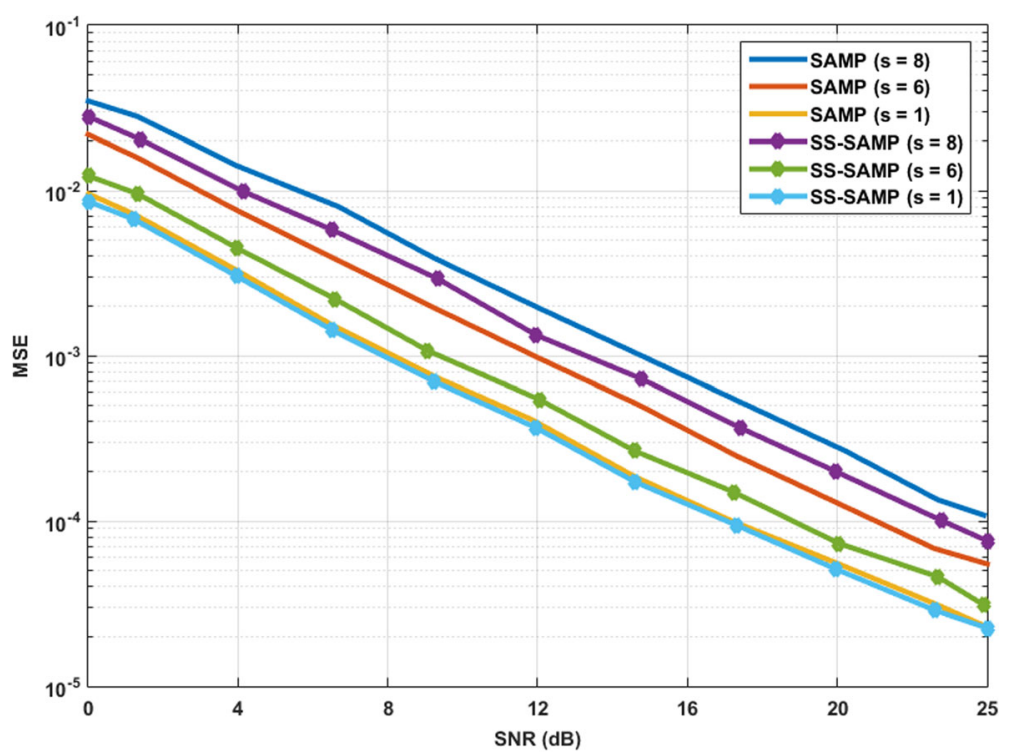

Fig. 4 MSE calculation of SAMP and SS-SAMP with different step sizes

obtained, it can be said that the proposed SS-SAMP can be used in a mobile computational limited environment with the computational complexity of slightly higher than SAMP.

As from the previous results, it can be seen that the proposed SS-SAMP is a good candidate for a mobile computational limited environment. It is also required to analyze the performance under different VLC system key parameters. Earlier in Section 4, the key parameters of VLC system were discussed and now the performance analysis is given. To start with the analysis, the key simulation parameters are the same as mentioned in
Table 1 . The first sets of parameter that will be analyzed are the LED nonlinearity and the effect of the shot noise and thermal noise on the VLC system. To investigate the output characteristics of a practical LED, an LED transfer function is modeled through a polynomial using the leastsquare curve fitting technique. An OPTEK white LED is considered in this simulation. For this type of LED, the forward current varies from $100 \mathrm{~mA}$ to $1 \mathrm{~A}$ and the output optical power lies between $90 \mathrm{~mW}$ to $400 \mathrm{~mW}$. Since according to the European lighting standard [44], the illuminance for home/office environments is $400 \mathrm{~lx}$. To check the LED nonlinearity, Fig. 6 shows the achievable

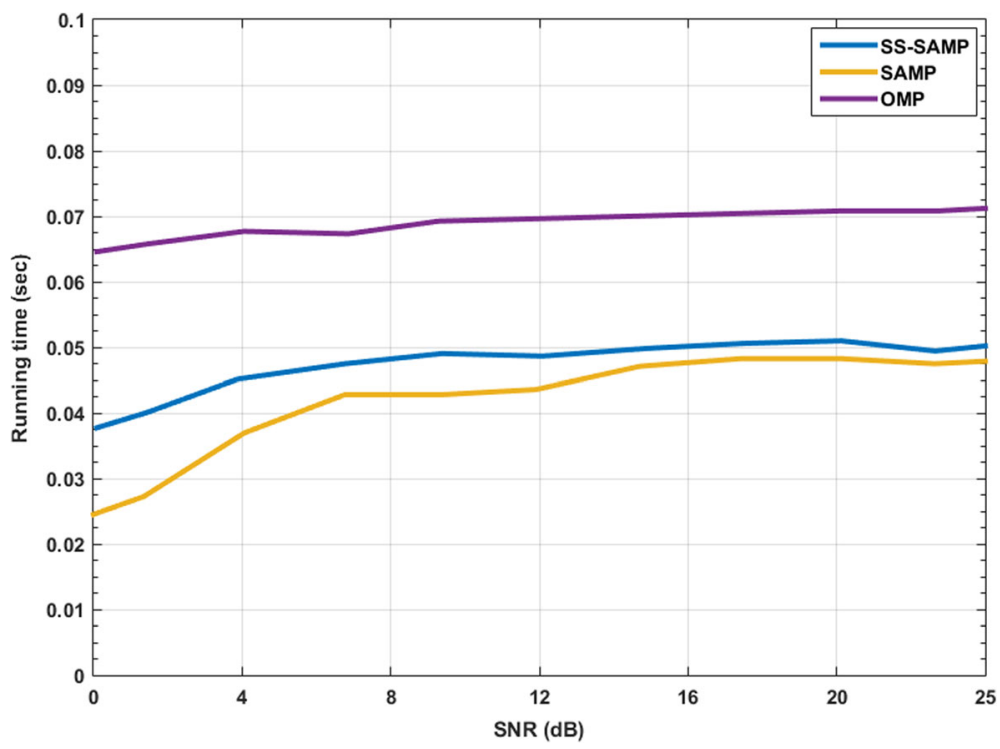

Fig. 5 Running time of different CS-based channel estimation algorithms 


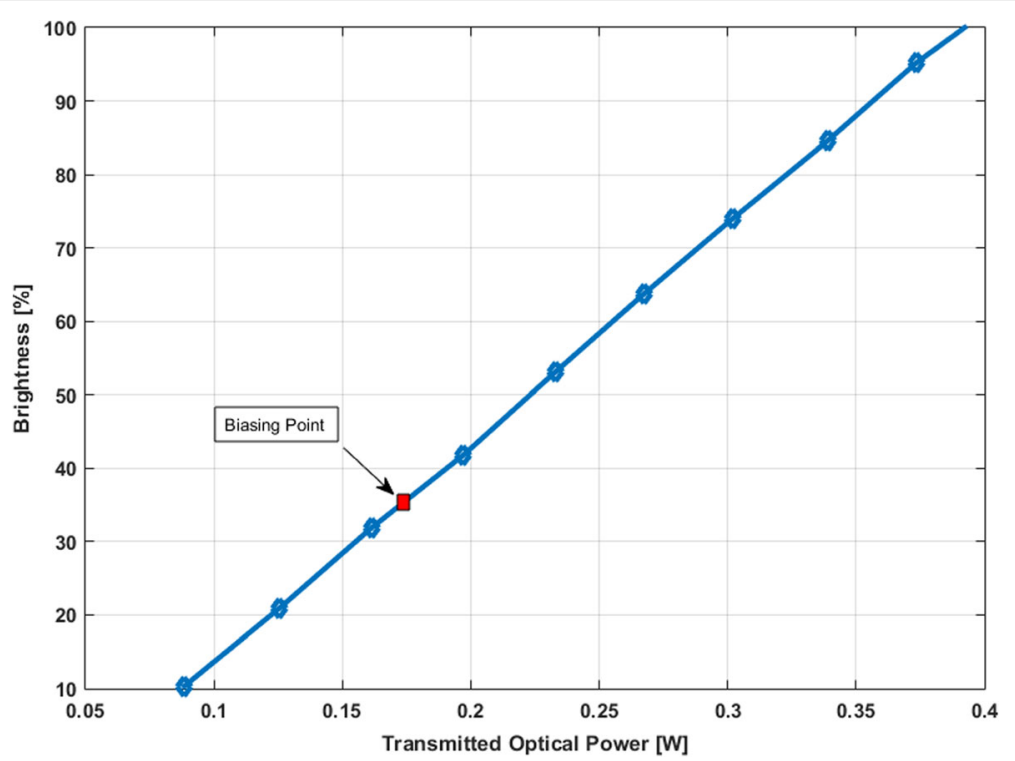

Fig. 6 Transmitted optical power vs. brightness of LED

brightness in percentage of a function of transmitted optical power of the given LED light. For this analysis, it is assumed that the dimming level is $0 \%$ and, to achieve the European lighting standard, the LED should operate at a minimum 35\% brightness level. To operate at this level, Fig. 6 depicts the recommended bias point of operation that is $(350 \mathrm{~mA} / 180 \mathrm{~mW})$.

The performance of the system is assessed in terms of average SNR at the receiver. The SNR values obtained were simulated against the transmitted optical power of both ideal and practical LED. Figure 7 shows the SNR of a practical LED operated at different biasing values, while Fig. 8 shows the performance of an ideal LED under the same biasing values. It is assumed that the modulation bandwidth is of $20 \mathrm{MHz}$. On observation, it can be seen that the system performance improves as the optical power is increased. At the recommended biasing point, the system shows the best performance. If the optical power is increased beyond this point the system performance starts to deteriorate. This is due to the nonlinearity distortion induced by the dynamic range of the LED. At this point, the signal clipping is more highlighted and the clipping noise becomes significant. One major point to note is that by setting the proper

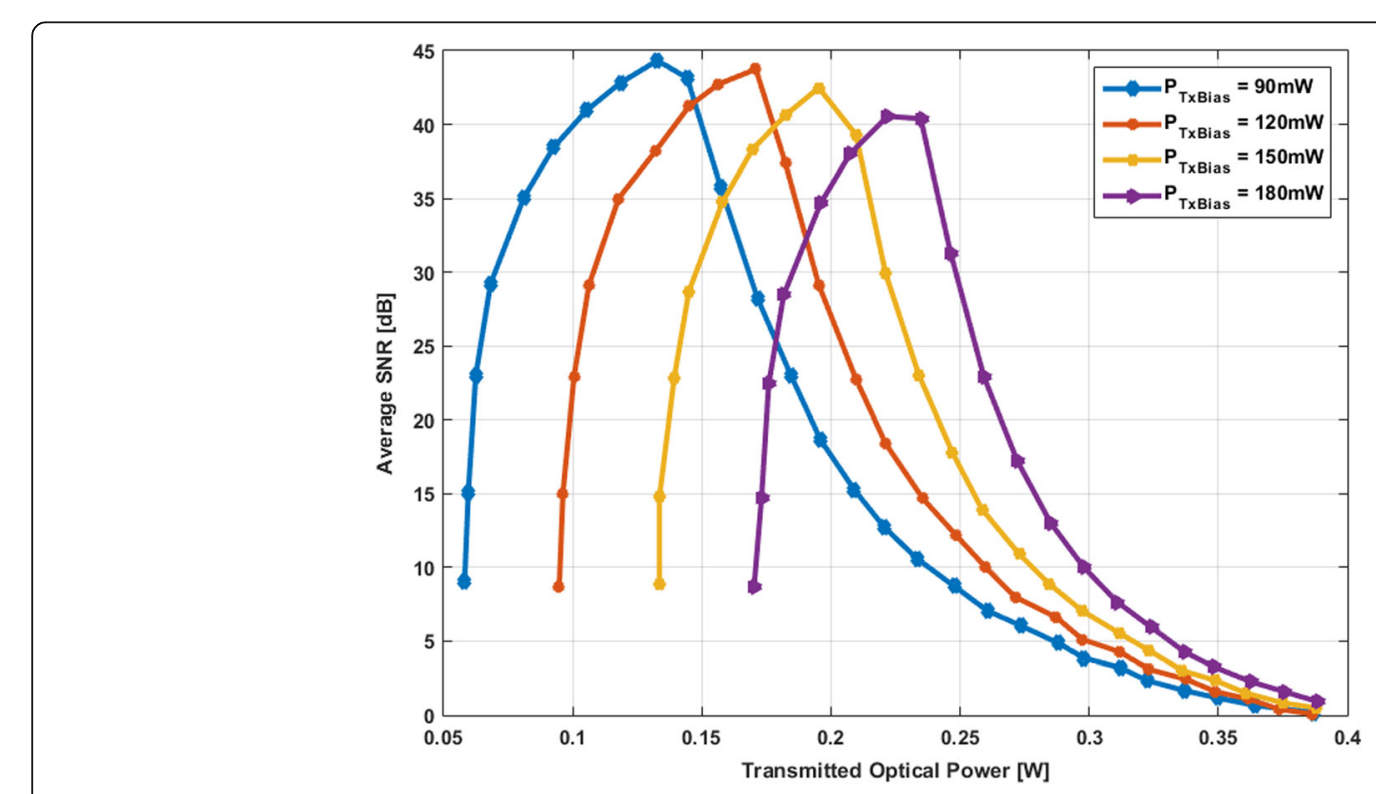

Fig. 7 Average SNR of a practical LED 


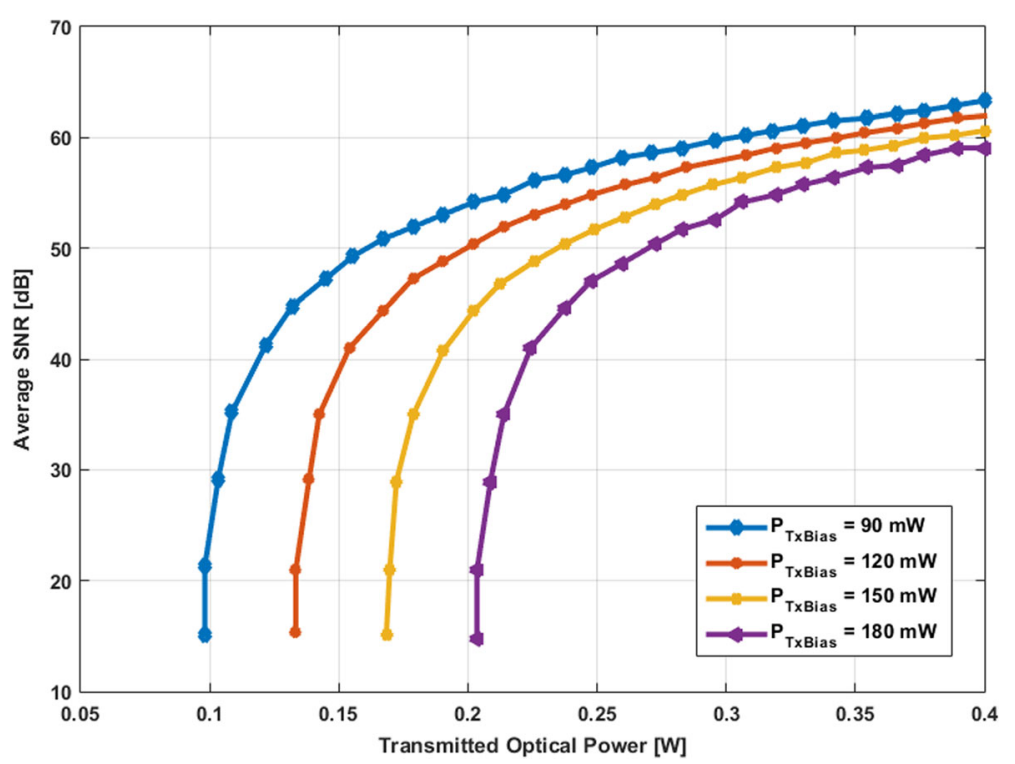

Fig. 8 Average SNR of an ideal LED

biasing point, the best SNR can be achieved for the VLC system. As an example, if the system wants to operate at $35 \%$ brightness to maintain $400 \mathrm{~lx}$, the best SNR value is $44 \mathrm{~dB}$ which is achieved if the biasing power is set to $120 \mathrm{~mW}$ and the optical signal power is $60 \mathrm{~mW}$. For the same brightness but at a lower SNR of $38 \mathrm{~dB}$, the biasing power should be $150 \mathrm{~mW}$ and the optical power at $30 \mathrm{~mW}$. Multiple configuration can be obtained depending on what SNR is required for the VLC system to operate efficiently. One thing to note is that whatever the biasing power and optical power is selected, the resulted optical transmitted power will always be $180 \mathrm{~mW}$ in this specific case. Since this simulation only deals with the minimum illumination requirements, other possible operating range can be determined by the method and relevant SNR can be computed.

The channel response of the diffused environment which includes both LOS and NLOS signals can be seen in Fig. 9. The channel gain is given in Eq. (6), and the optical wireless channel model is already mentioned at the start of this section. From the figure, it can be clearly seen that the main LOS component and the additional reflected NLOS components and their relevant reflection count.



Fig. 9 Channel response 


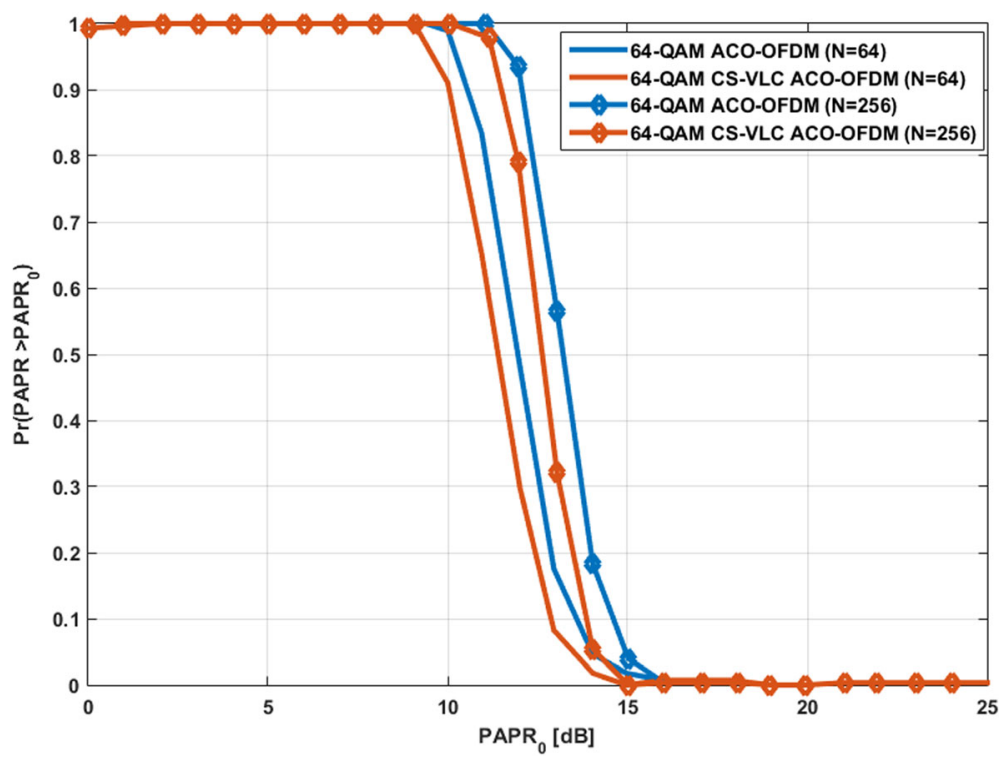

Fig. 10 PAPR calculation

The next variable to investigate is the PAPR of the proposed VLC system. The PAPR calculation again follow the same simulation parameters with the results averaging of 1000 Monte-Carlo simulations. The carrier size $N$ is changed from $N=64$ to $N=256$, and the comparison is made based on the performance. Also, PAPR of a simple ACO-OFDM VLC system is compared with the proposed CS ACO-OFDM VLC system. There is a slight improvement of using the CS-based methods because CS exploits the sparsity of the signal. Since in ACO-OFDM, only odd sub-carriers are sent and then applying CS techniques, the data size is further reduced and that can help improve the PAPR of the system. Figure 10 shows the CCDF of the PAPR for both normal ACO-OFDM VLC and CS-based ACO-OFDM VLC. It can be seen that the PAPR is slightly improved but as the number of sub-carriers are increased from 64 to 256 the PAPR is increased.
Since ACO-OFDM performs better with lower modulation levels and less sub-carriers, it is safe to use CSbased techniques for limited computational mobile based application.

The constellation diagrams of a simple ACO-OFDM and proposed CS-based ACO-OFDM system are analyzed next. Figure 11 shows the constellation diagram of both implementation side by side. Figure 11a shows the constellation diagram of a 64-QAM ACO-OFDM while Fig. 11b shows the constellation diagram of a 64-QAM CS-based ACO-OFDM. The constellation diagram of Fig. 11a is fuzzy as it is degraded by the effect of channel, and the BER performance is degraded as there is interference among different constellation points. As CS-based channel estimation techniques are used, the overall constellation is improved and every constellation point can be identified easily. This will also improve the BER performance of the VLC system.
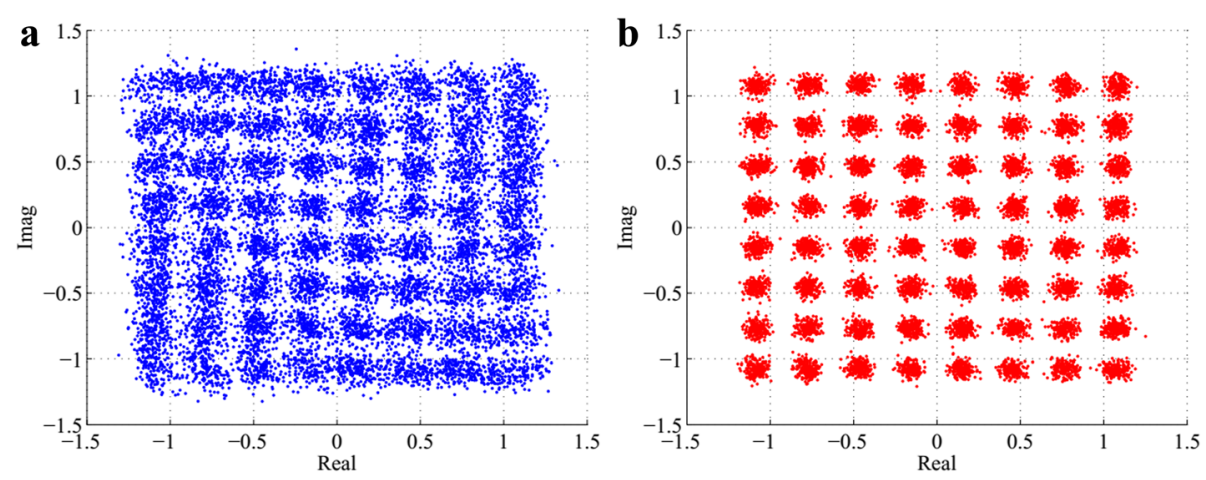

Fig. 11 Constellation diagrams. a Constellation of simple ACO-OFDM VLC. b Constellation of CS-based ACO-OFDM VLC 


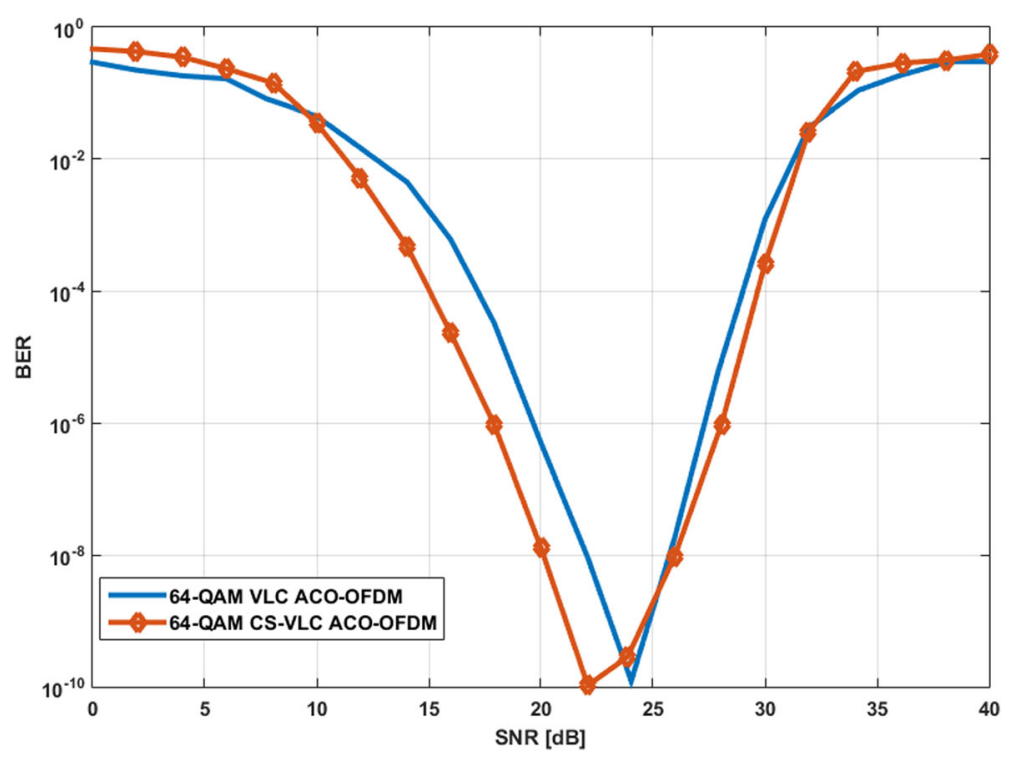

Fig. 12 BER comparison

To conclude the analysis of VLC system key parameters, the last parameter to look into is of BER performance. Figure 12 shows the overall performance of both simple ACO-OFDM and proposed CS-based ACO-OFDM systems. It can be seen that the CS-based ACO-OFDM performs better. The important thing to mention here is the effect of the LED nonlinearity. If the biasing point is not chosen carefully, the system performance will degrade drastically. Figure 12 is based on the values chosen in the previous mentioned analysis.

\section{Conclusions}

In this paper, a new modified CS-based channel estimation algorithm SS-SAMP was proposed for the ACOOFDM VLC system. The performance was evaluated in terms of BER, MSE, computational complexity, and key parameters of VLC system (nonlinearity, noise, channel response). The results show that CS-based techniques: OMP, SAMP, and SS-SAMP perform better than the traditional LS-based method. SS-SAMP stood out to be the best among the algorithms applied to the VLC-based system. The OMP algorithm requires the knowledge of sparsity beforehand. The SAMP algorithm is an improved adaptive version of OMP, but the computational cost is on the higher side due to the fact that the SAMP algorithm has to start with a random step size. It is shown through the performance analysis that SS-SAMP can improve the channel estimation accuracy without significantly increasing the computational complexity. It can be said that CS-technology-based algorithms can be used with the ACO-OFDM VLC system and can give accurate estimation of the original signal and still can manage to have an acceptable computation time.

\section{Acknowledgements}

This work was supported by the National Research Foundation of Korea (NRF) grant funded by the Korea government (No. 2016R1A2B4008457) and by the Strengthening R \& D Capability Program of Sejong University.

\section{Authors' contributions}

The research presented in this paper was a collaborative effort among all authors. MTN conceived, implemented and simulated the results along with the paper writeup. FI and WE wrote the paper and discussed the results. HSK revised the manuscript critically. All authors read and approved the final manuscript.

\section{Competing interests}

The authors declare that they have no competing interests.

\section{Author details}

'Department of Information and Communication Engineering, Sejong University, Gwangjin-gu, Seoul, Republic of Korea. ${ }^{2}$ Department of Electrical and Computer Engineering, Ryerson University, Toronto, Ontario, Canada.

Received: 29 April 2016 Accepted: 14 November 2016 Published online: 25 November 2016

\section{References}

1. Z Ghassemlooy, W Popoola, S Rajbhandari, Optical wireless communications: system and channel modelling with MATLAB ${ }^{\oplus}$. (CRC Press, 2012)

2. Qualcomm incorporated: the Qualcomm 1000x challenge (2013), HYPERLINK "https://www.qualcomm.com/invention/1000x" https://www. qualcomm.com/invention/1000x. Accessed 24 Apr 2016

3. MB Rahaim, L TDC, Towards practical integration of dual-use VLC within $5 G$ networks. IEEE Wirel Commun. 22, 97-103 (2015)

4. L Garber, Turning on the lights for wireless communications. Computer 44, 11-14 (2011)

5. H Elgala, R Mesleh, H Hass, Indoor optical wireless communication: potential and state-of-the-art. IEEE Commun. Mag. 49, 56-62 (2011)

6. DC O'Brien, L Zeng, H Le-Minh, G Faulkner, JW Walewski, S Randel, In IEEE 19th International Symposium on Personal, Indoor and Mobile Radio Communications, 2008. PIMRC 2008. Visible light communications: Challenges and possibilities, (2008), pp. 1-5.

7. J Armstrong, RJ Green, MD Higgins, Comparison of three receiver designs for optical wireless communications using white LEDs. IEEE Commun Lett 16, 748-751 (2012) 
8. J Armstrong, OFDM for optical communications. J Light Technol 27, 189-204 (2009)

9. SD Dissanayake, J Armstrong, Comparison of ACO-OFDM, DCO-OFDM and ADO-OFDM in IM/DD systems. J Light Technol 31, 1063-1072 (2013)

10. JM Kahn, JR Barry, , Wireless infrared communications. Proc IEEE 85, 265-298 (1997)

11. J Armstrong, AJ Lowery, Power efficient optical OFDM. Electron Lett 42, 370-372 (2006)

12. P Errong, C Fang, L Jibi, Optimal power allocation for OFDM-based cognitive radio systems with fair protection against primary users. J Comput Inf Syst 8, 9831-9837 (2012)

13. D Wu, Z Wang, R Wang, J He, Q Zuo, H Zhao, Channel estimation for asymmetrically clipped optical orthogonal frequency division multiplexing optical wireless communications. IET Commun 6, 532-540 (2012)

14. H Zhao, M Li, R Wang, D Wu, Channel estimation for asymmetrically clipped optical orthogonal frequency division multiplexing communication system. Opt Eng 52, 076111 (2013)

15. S Qaisar, RM Bilal, W lqbal, M Naureen, S Lee, Compressive sensing: from theory to applications, a survey. J Commun Netw 15, 443-456 (2013)

16. EJ Candes, MB Wakin, An introduction to compressive sampling. IEEE Signal Proc Mag 25, 21-30 (2008)

17. $S \mathrm{Ji}, \mathrm{Y} X \mathrm{Xue}, \mathrm{L}$ Carin, Bayesian compressive sensing. IEEE Trans Signal Proc $56,2346-2356(2008)$

18. EJ Candes, J Romberg, Sparsity and incoherence in compressive sampling. Inverse Probl 23, 969 (2007)

19. EJ Candes, The restricted isometry property and its implications for compressed sensing. C. R. Math 346, 589-592 (2008)

20. EJ Candes, J Romberg, T Tao, Robust uncertainty principles: exact signal reconstruction from highly incomplete frequency information. IEEE Trans Inf Theory 52, 489-509 (2006)

21. YY Won, SM Yoon, Compressive sensing-based channel bandwidth improvement in optical wireless orthogonal frequency division multiplexing link using visible light emitting diode. Optics Express 22, 19990 (2014)

22. J Tropp, A Gilbert, Signal recovery from random measurment via orthogonal matching pursuit. IEEE Trans Inf Theory 53, 4655-4666 (2007)

23. C Berger, S Zhou, J Preisig, P Willett, Sparse channel estimation for multicarrier underwater acoustic communication: from subspace methods to compressed sensing. IEEE Trans Signal Proc 58, 1708-1721 (2010)

24. C Berger, Z Wang, J Huang, S Zhou, Aplication of compressive sensing to sparse channel estimation. IEEE Commun Mag 48, 164-174 (2010)

25. T Do, NNG Lu, T Tran, Proceedings of the 42nd Asilomar Conference on Signals, Systems and Computer (Sparsity adaptive matching pursuit algorithm for practical compressed sensing, Pacific Grove, CA, 2008), pp. 581-587

26. CN Reinhardt, S Jaruwatanadilok, Y Kuga, A Ishimaru, JA Ritcey, Investigation of multilevel amplitude modulation for a dual-wavelength free-space optical communications system using realistic channel estimation and minimum mean-squared-error linear equalization. Appl. Opt 47, 5378-5389 (2008)

27. S Liu, F Yang, J Song, Narrowband interference cancelation based on priori aided compressive sensing for DTMB systems. IEEE Trans Broadcast 61(1), 66-74 (2015)

28. AJC Moreira, RT Valadas, AM Oliveira Duarte, IET Conference Proceedings, Reducing the Effects of Artificial Light Interference in Wireless Infrared Transmission Systems, 1996, p. 5

29. RP Jimenez, J Rabadan, FL Hernandez, Filtered modulation schemes for short distance infrared wireless communications. IEEE Trans Consum Electron 46(2), 275-282 (2000)

30. F Delgado, RP Jiménez, J Rabadán, FL Hernández, Design of fast frequencyhopping spread-spectrum system for wireless infrared communications. Electron Lett 36(17), 1510 (2000)

31. J Rabadan, M Bacallado, F Delgado, S Perez, RP Jimenez, Experimental characterization of a direct-sequence spread-spectrum optical wireless system based on pulse-conformation techniques for in-house communications. IEEE Trans Consum Electron 50(2), 484-490 (2004)

32. O Gonzalez, RP Jimenez, S Rodriguez, J Rabadan, A Ayala, OFDM over indoor wireless optical channel. IEE Proc - Optoelectron 152(4), 199 (2005)

33. J Barry, Wireless Infrared Communications (Kluwer Academic, Boston, MA, (2004)

34. R Gagliardi, S Karp, Optical Communications (Wiley, New York, 1984)

35. M Wang, J Wu, W Yu, H Wang, J Li, J Shi, C Luo, Efficient coding modulation and seamless rate adaptation for visible light communications. IEEE Wirel Commun 22(2), 86-93 (2015)
36. C Berger, Z Shengli, J Preisig, P Willett, Sparse channel estimation for multicarrier underwater acoustic communication: from subspace methods to compressed sensing. IEEE Trans Signal Proc 58(3), 1708-1721 (2010)

37. W Li, J Preisig, Estimation of rapidly time-varying sparse channels. IEEE J Ocean Eng 32(4), 927-939 (2007)

38. W Bajwa, J Haupt, A Sayeed, R Nowak, Compressed channel sensing: a new approach to estimating sparse multipath channels. Proc IEEE 98(6), 1058-1076 (2010)

39. S Cotter, B Rao, Sparse channel estimation via matching pursuit with application to equalization. IEEE Trans Commun 50(3), 374-377 (2002)

40. R Prasad, C Murthy, B Rao, Joint approximately sparse channel estimation and data detection in OFDM systems using sparse Bayesian learning. IEEE Trans Signal Proc 62(14), 3591-3603 (2014)

41. H Elgala, R Mesleh, H Haas, Indoor broadcasting via white LEDs and OFDM. IEEE Trans Consum Electron 55, 1127-1134 (2009)

42. O Gonzalez, RP Jimenez, A Ayala, OFDM over indoor wireless optical channel. IEE Proc - Optoelectron 152, 199-204 (2005)

43. L Weihao, Z Difan, X Zhengyuan, Modelling of optical wireless scattering communication channels over broad spectra. J Opt Soc Am A 32, 486-490 (2015)

44. European Standard EN 12464-1, Lighting of indoor Work Places, 2009

\section{Submit your manuscript to a SpringerOpen ${ }^{\circ}$ journal and benefit from:}

- Convenient online submission

- Rigorous peer review

- Immediate publication on acceptance

- Open access: articles freely available online

- High visibility within the field

- Retaining the copyright to your article

Submit your next manuscript at $\gg$ springeropen.com 